Pacific Journal of Mathematics

A GENERALIZATION OF SEPARABLE GROUPS 


\title{
A GENERALIZATION OF SEPARABLE GROUPS
}

\author{
E. F. CoRnelius, JR.
}

\begin{abstract}
This paper introduces a new class of torsion free abelian groups, the class of quasi-separable groups, which is the quasi-isomorphism analog of the class of separable groups and which properly contains the latter. Our purpose is two-fold: first, to further explore the phenomena of quasi-isomorphism, which has proved fruitful in the study of torsion free groups, and second, to shed further light on separable groups.
\end{abstract}

The term "group" herein refers to a torsion free abelian group. As is customary when dealing with quasi-isomorphism, we assume that all groups are subgroups of a fixed vector space $V$ over the rational number field $Q . L(V)$ denotes the algebra of linear transformations of $V . L(V)$ is equipped with the finite topology [7] throughout and topological terms refer to this topology unless otherwise stated. $G$ always denotes a full subgroup of $V$, i.e., a subgroup with torsion quotient $V / G ; G$ is full in $V$ if and only if $V$ is its unique minimal divisible extension. $Q E(G)$ is the quasi-endomorphism algebra of $G$ and $Q F(G)$ is the ideal of $Q E(G)$ consisting of elements of finite rank.

Our approach is to recall that there is a one-to-one correspondence between quasi-decompositions of a group $G$ and idempotents in $Q E(G)$ [8]. Thus a group with "many" quasi-decompositions has "many" quasi-endomorphisms of a particular type. In §1, quasi-separable groups are defined and basic properties are explored. A principal result is that every pure subgroup of finite rank in $G$ is a quasisummand of $G$ if and only if $G$ is quasi-separable with linearly ordered type set, $T(G)$. In $\$ 2$, a characterization of homogeneous quasiseparable groups is obtained, namely, $G$ is homogeneous and quasiseparable if and only if $Q F(G)$ is dense in the finite topology of $L(V)$. In $\S 3$, attention focuses on separable groups. It is shown that a countable group $G$ is homogeneous and completely decomposable if and only if $Q E(G)$ is dense. Finally, a description of homogeneous separable groups is obtained in terms of their endomorphisms. For example, a countable group $G$ is homogeneous and completely decomposable if and only if for any pair of independent elements $a_{1}, a_{2}$ in $G$ and any arbitrary pair of elements $b_{1}, b_{2}$ in $G$, there exists an endomorphism $f$ of $G$ such that $f a_{i}=n b_{i}, i=1,2, n$ some positive integer.

General abelian group theory [5] is assumed. By this date, quasi-isomorphism is a familiar concept of this theory so basic facts 
are used here without comment; a complete background may be obtained from $[1,2,8,9] . \doteq$ and $\doteq$ denote quasi-contained and quasi-equal, respectively. Recall that $Q E(G)=\{f \in L(V): f G \leqq G\}$. Each endomorphism of $G$ has a unique extension to a linear transformation of $V$ and we use the same symbol to denote both. $h(a)$ denotes the height of the element $a$; if it is not clear from context in which group height is computed, a subscript is appended, e.g., $h_{G}(a)$. Similarly, $t(a)$ denotes the type of the element $a ; t(H)$ may also denote the type of a homogeneous group $H$. Notation is abused for the sake of conciseness; e.g., the same symbol $Z$ is used to denote both the ring of integers and its additive group. $S^{*}$ denotes the subspace spanned by the subset $S$ of $V$; it is also used to denote the subalgebra generated by a subset of $L(V)$. All sums are direct; e.g., notation such as $G \doteq A+B$ implies that $A$ and $B$ are disjoint subgroups of $V$ and we call $A$ a quasi-summand of $G$. Additional notation is introduced as needed.

\subsection{Quasi-separable groups.}

DeFinition 1.1. Call a group $G$ quasi-separable if and only if every finite subset of $G$ is contained in a completely decomposable quasi-summand.

REMark 1.2. Suppose $G$ is quasi-separable and suppose $F$ is a finite subset of $G$; by definition $G \doteq A+B$ for some groups $A$ and $B$ contained in $V$, with $A$ completely decomposable and containing $F$. Clearly $A$ may be assumed to have finite rank without any loss of generality. Now $G \doteq A \cap G+B \cap G$ and $F \subseteq A \cap G$, but $A \cap G$ need not be completely decomposable even if $A$ has finite rank; see for example Lemma 9.3 [2]. However, if $A$ has finite rank and $T(A)$ is linearly ordered (especially $\mathrm{f} A$ is homogeneous), then $A \cap G$ is also completely decomposable by Corollary 9.6 [1]. Thus if $T(G)$ is linearly ordered, $A$ may be assumed to be a completely decomposable, pure subgroup [1, p. 95] of finite rank in $G$.

The following modular law will prove indispensable.

Proposition 1.3. Suppose $H \subseteq A+B$ and $A \subseteq H$ for groups $H$, $A$, and $B$. Then $H \doteq A+H \cap B$.

Proof. For some positive integer $n, n A \subseteq H$ so

$$
n(A+H \cap B) \subseteq H .
$$

If $m H \subseteq A+B$ for $m$ a positive integer, then $n m H \subseteq n A+n B$; 
i.e., for $c \in H$, mnc may be written $m n c=n a+n b$ with $a \in A$ and $b \in B$. Now $n b=m n c-n a \in H \cap B$ so $m n H \subseteq A+H \cap B$.

REMARK 1.4. Let $n$ be a positive integer. Consider a group having the property: (1) every pure subgroup of rank $n$ is a quasisummand. It is easy to see that every pure subgroup of rank $n$ is a quasi-summand of $G$ if and only if $Q E(G)$ contains a projection onto any $n$-dimensional subspace of $V$. Consequently if $G$ has property (1), so does any quasi-summand of $G$. Also, by Proposition 1.3, if $G$ satisfies (1), so does any pure subgroup of $G$. Corresponding results hold for the property: (2) every pure subgroup is a quasi-summand.

LEMMA 1.5. If every pure subgroup of rank one is a quasisummand of $G$, then every pure subgroup of finite rank is a quasisummand which is quasi-equal to a completely decomposable group.

Proof. Assume the result for pure subgroups of rank $\leqq n$ and let $H$ be a pure subgroup of rank $n+1 \geqq 2$. Let $A \subset H$ be pure of rank $n$; by hypothesis $G \doteq A+B$ with $A$ quasi-equal to a completely decomposable group; take $B$ pure in $G[1, \mathrm{p} .95]$. By Proposition 1.3, $H \doteq A+H \cap B$; clearly $H \cap B$ is a pure subgroup of rank one in $B$. By Remark 1.4, $B \doteq H \cap B+C$ and so $G \doteq A+H \cap B+C \doteq H+C$, which completes the proof.

We shall shortly be able to strengthen the conclusion of Lemma 1.5 (see Corollary 1.7). A complete description of groups with the property that every pure subgroup of finite rank is a quasi-summand can be obtained from the following theorem, which is the quasiisomorphism analog of Theorem 46.8 [5].

THEOREM 1.6. Every pure subgroup of $G$ is a quasi-summand if and only if $G=D+G_{1}+\cdots+G_{n}$ with $D$ divisible and the $G_{i}$ reduced rank-one groups satisfying $t\left(G_{1}\right) \leqq \cdots \leqq t\left(G_{n}\right)$.

Proof. Suppose $G$ has the property that every pure subgroup is a quasi-summand and write $G==D+H$ with $D$ divisible and $H$ reduced; by Remark 1.4, $H$ inherits this property. To see that $H$ has finite rank, suppose $\left\{a_{i}\right\}_{i=1}^{\infty}$ is an independent set in $H$. Let $A$ be the pure subgroup of $H$ generated by $\left\{a_{i}-(i+1) a_{i+1}\right\}_{i=1}^{\infty} ; a_{1} \notin A$. Now $H / A$ contains a divisible subgroup generated by $\left\{a_{i}+A\right\}_{i=1}^{\infty}$, so $A$ could not be a quasi-summand of the reduced group $H[2, \mathrm{p} .26]$. Thus $H$ has finite rank and by Lemma $1.5, H \doteq H_{1}+\cdots+H_{n}$ with $H_{i}$ of rank one, $i=1, \cdots, n$. It will be sufficient to show that the types of any two of the $H_{i}$ are comparable, for then a suitable 
relabeling of the $H_{i}$ and Corollary 9.6 [1] will complete the proof. Let $B$ and $C$ be distinct among the $H_{i}$; by Remark 1.4, every pure subgroup of $B+C$ is a quasi-summand since $B+C$ is a quasi-summand of $H$. Suppose the types of $B$ and $C$ are incomparable; then $B+C$ contains elements of three different types, $t(B), t(C)$, and $t(B) \cap t(C)$. Pick nonzero elements $b$ and $c$ of $B$ and $C$, respectively, and let $M$ be the pure subgroup of $B+C$ generated by $b+c$. But $B+C \doteq M+N$ is impossible because $M+N$ cannot contain both elements of type $t(B)$ and of type $t(C)$, since $t(M)=t(B) \cap t(C)$ [2, p. 26]. This contradiction shows that $t(B)$ and $t(C)$ are in fact comparable and so completes the first half of the proof. Conversely suppose $G=D+H$ with $D$ divisible, $H=G_{1}+\cdots+G_{n}$, and the $G_{i}$ reduced rank-one groups satisfying $t\left(G_{1}\right) \leqq \cdots \leqq t\left(G_{n}\right)$. First, to see that it will be sufficient to treat the case $D=0$, recall that any pure subgroup $A$ of $G$ decomposes into $A=B+C$ with $B$ divisible and $C$ reduced and that $D \cap C=0$ because $C$ is pure in $G$. Thus the complement $H$ of $D$ may be chosen to contain $C[5$, p. 63]. Since $B$ is a direct summand of $D$, it will be enough to show that $C$ is a quasi-summand of $H$, so we assume $D=0$. By Remark 1.4 and Lemma 1.5, it will be sufficient to show that $Q E(G)$ contains a projection onto any one-dimensional subspace of $V$. Let $x \in V$ be nonzero; $k x \in G$ for some positive integer $k$ and so $k x=a_{1}+\cdots+a_{n}$ with $a_{i} \in G_{i}, i=1, \cdots, n$. Let $a_{j}$ be the first nonzero $a_{i}$;

$$
t_{G}(k x)=t_{G}\left(a_{j}\right)=t\left(G_{j}\right) \text {. }
$$

If $S$ denotes the pure subgroup of $G$ generated by $k x$, then $G_{j}$ is isomorphic to $S$ via some map $f$. Since $G_{j}$ has rank one, for some non-zero integers $r$ and $s, r f^{-1}(k x)=s a_{j}$. If $g$ denotes the map from $G$ onto $S \subseteq G$ induced by $f$, then $(s / r) g \in Q E(G)$ projects $V$ onto the subspace spanned by $x$.

COROLLARY 1.7. These properties of a group $G$ are equivalent: (1) Every pure subgroup of rank one in $G$ is a quasi-summand; (2) every pure subgroup of finite rank in $G$ is a completely decomposable quasi-summand; (3) $G$ is quasi-separable with linearly ordered type set.

Proof. Assume (1) is true and let $S$ be a pure subgroup of finite rank in $G$. By Lemma 1.5, $S$ is a quasi-summand of $G$ and thus by Remark 1.4, every pure subgroup of $S$ is a quasi-summand of $S$. Theorem 1.6 shows that $S$ is completely decomposable with linearly ordered type set. Thus we have (1) implies (2) and (2) implies (3). Finally, suppose (3) holds and let $H$ be a pure subgroup of rank one 
in $G$. By Remark 1.2, $H$ is contained in a pure subgroup $S$ of $G$ which is a completely decomposable group of finite rank with linearly ordered type set. By Theorem 1.6, $H$ is a quasi-summand of $S$ and thus of $G$.

From the foregoing results it is perhaps clear that a quasiseparable group need not be separable; a specific example is the following. It is well known that the subgroup $S$ of $\pi=\prod_{i=1}^{\infty} Z$ generated by $2 \pi$ and $\Sigma=\sum_{i=1}^{\infty} Z \subseteq \pi$ is not separable. Since $S \doteq \pi$, both groups have the same quasi-endomorphism algebra [8]. It is also known that $\pi$ is homogeneous and separable, so by Theorem $2.5, S$ is quasiseparable. In fact, there exist rank-two groups which are quasiseparable but not separable, i.e., not the direct sum of two rank-one groups; see for example Lemma 9.3 [2].

Just as for separable groups, the direct sum of a collection of quasi-separable groups is quasi-separable and the tensor product of two quasi-separable groups is quasi-separable.

Having proved basic results about quasi-separable groups, we turn our attention to the homogeneous case.

2.0. Homogeneous quasi-separable groups. We proceed to obtain a characterization of homogeneous quasi-separable groups in terms of quasi-endomorphisms. Intuitively, a group is homogeneous and quasi-separable precisely when it has "enough" quasi-endomorphisms; this is formulated in terms of density in the finite topology [7] of $L(V)$.

Recall [9] that a group is irreducible if and only if it has no nontrivial, pure, fully invariant subgroups, that an irreducible group is homogeneous, and that $G$ is an irreducible group if and only if $V$ is an irreducible $Q E(G)$-module. After Jacobson [7], call a subset $S$ of $L(V) k$-fold transitive if and only if given any $j \leqq k$ linearly independent vectors $x_{1}, \cdots, x_{j}$ in $V$ and any $j$ vectors $y_{1}, \cdots, y_{j}$ in $V$, there exists $f \in S$ such that $f x_{i}=y_{i}, i=1, \cdots, j$. Note well that $G$ is irreducible, and thus homogeneous, if and only if $Q E(G)$ is onefold transitive.

REMARK 2.1. For a subring $R$ of $L(V)$ the following conditions are equivalent: (1) $R$ is two-fold transitive; (2) $R$ is $k$-fold transitive for every $k$; (3) $R$ is dense in $L(V)$. This follows immediately from Jacobson [7, p. 32].

LEMma 2.2. Let $H$ be a pure subgroup of $G$ and let $f$ be any quasi-endomorphism of $G$ such that $f\left(H^{*}\right) \subseteq H^{*}$. Then the restriction of $f$ to $H^{*}$ is a quasi-endomorphism of $H$. 
Proof. Let $n$ be a positive integer such that $n(f G) \subseteq G$; then

$$
n(f H) \subseteq G \cap(f H)^{*} \sqsubseteq G \cap\left(H^{*}\right)=H .
$$

Proposition 2.3. (1) $Q E(G)$ is dense if and only if $G$ is irreducible and $Q$ is the centralizer of $Q E(G)$ in $L(V)$.

(2) If $Q E(G)$ is dense, then $G$ is homogeneous and every pure subgroup of finite rank in $G$ is completely decomposable.

(3) $Q F(G)$ is an ideal of $Q E(G)$; if $Q E(G)$ is dense and $Q F(G) \neq 0$, then $Q F(G)$ is also dense.

Proof. (1) follows from a remark of Jacobson [7, p.32] and the fact that $G$ is irreducible if and only if $V$ is an irreducible $Q E(G)$ module. Let $H$ be a pure subgroup of finite rank in $G$. In order to prove (2), it will suffice to show that $Q E(H)=L\left(H^{*}\right)$ by Corollary 1.5 [4]. Let $x_{1}, \cdots, x_{n}$ be a basis of $H^{*}$ and let $f \in L\left(H^{*}\right)$. By density and Remark 2.1, some $g \in Q E(G)$ maps $x_{i}$ to $f x_{i}, i=1, \cdots, n$, and so $g\left(H^{*}\right) \subseteq H^{*}$. By Lemma 2.2, $g$ restricted to $H^{*}$ is a quasiendomorphism of $H$ and so $Q E(H)=L\left(H^{*}\right)$. In (3), it is clear that $Q F(G)$ is an ideal of $Q E(G)$; Theorem 4 [7, p.33] completes the proof.

LEMMA 2.4. If $Q F(G)$ is dense, then it contains a projection onto any finite dimensional subspace of $V$ and thus every pure subgroup of finite rank in $G$ is a completely decomposable quasi-summand.

Proof. Let $x_{1}, \cdots, x_{n}$ be independent in $V$. By density and Remark 2.1, some $f \in Q F(G)$ leaves the $x_{i}$ invariant. Extend $x_{1}, \cdots, x_{n}$ to a basis $x_{1}, \cdots, x_{n}, y_{1}, \cdots, y_{m}$ of $f V$. Again, some $g \in Q F(G)$ leaves the $x_{i}$ invariant and annihilates $y_{1}, \cdots, y_{m}$. Now $g f$ projects $V$ onto the subspace spanned by $x_{1}, \cdots, x_{n}$. Suppose $H$ is a pure subgroup of finite rank in $G$; by (2) of Proposition 2.3, $H$ is completely decomposable. We have just proved that $Q F(G)$ contains a projection $e$ of $V$ onto $H^{*}$. Now $G \doteq e V \cap G+(1-e) V \cap G$ and $e V \cap G=H$ because $H$ is pure.

We are now prepared to prove

THEOREM 2.5. These are equivalent:

(1) $G$ is homogeneous and quasi-separable.

(2) $Q F(G)$ is dense in the finite topology of $L(V)$.

(3) $Q F(G)$ is one-fold transitive and every pure subgroup of finite rank in $G$ is completely decomposable.

Proof. (1) implies (2). By Remark 2.1, it will be sufficient to show that $Q F(G)$ is two-fold transitive. Let $x_{1}$ and $x_{2}$ be independent 
in $V$ and let $y_{1}$ and $y_{2}$ be arbitrary elements of $V$. Since $G$ is a full subgroup of $V$, there is some positive integer $n$ such that $n x_{1}, n x_{2}$, $n y_{1}$, and $n y_{2}$ are all in $G$; suppose these elements are contained in a completely decomposable quasi-summand, $H$, of finite rank. $H$ is homogeneous because $G$ is, so by Corollary 1.5 [4], $Q E(H)=L\left(H^{*}\right)$. If $e$ is an idempotent associated with $H, Q E(H)=e Q E(G) e$ [8], so if $f \in L\left(H^{*}\right)$ sends $x_{i}$ to $y_{i}, i=1,2, f$ is induced by ege for some $g \in Q E(G)$. Now ege $\in Q F(G)$, so $Q F(G)$ is two-fold transitive and thus dense.

That (2) implies (3) follows from Remark 2.1 and Proposition 2.3.

(3) implies (1). $G$ is certainly homogeneous because $Q E(G)$ is one-fold transitive. By Corollary 1.7 and Remark 1.4, it will suffice to prove that $Q E(G)$ contains a projection onto any one-dimensional subspace of $V$, so let $x$ be any nonzero element in $V$. By hypothesis some $f \in Q F(G)$ leaves $x$ invariant; $A=f V \cap G$ is pure of finite rank in $G$ and so is completely decomposable. $B=\{x\}^{*} \cap G$ is a direct summand of $A$ [5, p. 178]. If $g$ projects $A$ onto $B$, then $g f \in Q E(G)$ projects $V$ onto $\{x\}^{*}$.

Under the hypothesis of Theorem 2.5, $Q E(G)$ is primitive with socle $Q F(G)$ by the Structure Theorem [7, p. 75].

3.0. Applications to separable groups. Here we prove that countable groups $G$ with $Q E(G)$ dense in $L(V)$ are homogeneous and completely decomposable; this is accomplished with the aid of a generalization of Pontryagin's criterion for countable free groups. $k$-fold transitivity of quasi-endomorphisms is interpreted in terms of endomorphisms to provide further insight into homogeneous quasiseparable groups. This suggests properties of endomorphisms both necessary and sufficient for a group to be homogeneous and separable.

Lemma 3.1. A countable homogeneous group is completely decomposable if and only if each pure subgroup of finite rank is completely decomposable.

Proof. The necessity obtains by Theorem 46.6 [5]. For the sufficiency, let $\left\{a_{i}\right\}_{i=1}^{\infty}$ be an enumeration of a countable homogeneous group $G$, each of whose pure subgroups of finite rank is completely decomposable. Let $H_{n}$ denote the pure subgroup generated by $a_{1}, \cdots, a_{n}$ and set $G_{1}=H_{1}$. Then in general, $H_{n+1}=H_{n}+G_{n+1}$ [5, p.178] with $G_{n+1}$ either 0 or of rank one. Now $G=\sum_{n=1}^{\infty} G_{n}$.

THEOREM 3.2. A countable group $G$ is homogeneous and completely decomposable if and only if $Q E(G)$ is dense. 
Proof. The necessity follows from Theorem 2.5 and the sufficiency from Proposition 2.3 (2) and Lemma 3.1.

CoROLlaRy 3.3. A countable, homogeneous, quasi-separable group is completely decomposable.

Corollary 3.4. If $Q E(G)$ is dense then $G$ is $\aleph_{1}$-completely decomposable in the sense that every countable pure subgroup is completely decomposable.

The discussion now turns to an interpretation in terms of endomorphisms of some properties of quasi-endomorphisms encountered in §2. $E(G)$ denotes the endomorphism ring of $G$ and $F(G)$ denotes those endomorphisms of $G$ which have finite rank.

DEFInITION 3.5. A subset $S$ of $E(G)$ is called $k$-fold transitive if and only if given $j \leqq k$ independent elements $a_{1}, \cdots, a_{j}$ of $G$ and any $j$ elements $b_{1}, \cdots, b_{j}$ of $G$, there exists an endomorphism $f \in S$ and a positive integer $n$ such that $f a_{i}=n b_{i}, i=1, \cdots, j$.

Proposition 3.6. The pure subring $R$ of $E(G)$ is $k$-fold transitive if and only if $R^{*}(\subseteq L(V))$ is $k$-fold transitive.

Proof. A straightforward computation using the fact that $E(G)$ is full in $Q E(G)$ and using the one-to-one correspondence between pure subrings of $E(G)$ and subalgebras of $Q E(G)$ [5, p.271].

REMARK 3.7. The above implies the following; (5) is of particular interest.

(1) $G$ is irreducible if and only if $E(G)$ is one-fold transitive.

(2) $F(G)$ is a pure ideal of $E(G)$ and $F(G)^{*}=Q F(G)$.

(3) The pure subring $R$ of $E(G)$ is two-fold transitive if and only if $R^{*}$ is dense.

(4) $G$ is homogeneous and quasi-separable if and only if $F(G)$ is two-fold transitive.

(5) A countable group $G$ is homogeneous and completely decomposable if and only if $E(G)$ is two-fold transitive.

A property somewhat stronger than two-fold transitivity may be required of $F(G)$ to conclude that $G$ is homogeneous and separable.

Definition 3.8. Call a subset $S$ of $E(G)$ fully $k$-fold transitive if and only if $S$ is $k$-fold transitive and in addition for any nonzero elements $a$ and $b$ of $G$ such that $h(a) \leqq h(b)$, some $f \in S$ maps $a$ to $b$. 
LEMMA 3.9. Let $a$ and $b$ be nonzero elements of rank-one groups $A$ and $B$, respectively. Then some $f \in \operatorname{Hom}_{Z}(A, B)$ maps $a$ to $b$ if and only if $h_{A}(a) \leqq h_{B}(b)$.

Proof. Only the sufficiency need be checked and this can be done computationally by using the characterization of subgroups of $Q$ found in [3].

THEOREM 3.10. The following statements about the group $G$ are equivalent.

(1) $G$ is homogeneous and separable.

(2) $F(G)$ is fully two-fold transitive.

(3) $F(G)$ is fully one-fold transitive and every pure subgroup of finite rank in $G$ is completely decomposable.

(4) $G$ is homogeneous, every pure subgroup of finite rank is completely decomposable, and $F(G)$ is dense in the finite topology of $E(G)$.

Proof. We prove that (1) and (2) are equivalent, then (2) and (3), and finally (1) and (4).

(1) implies (2). By Remark $3.7(4), F(G)$ is two-fold transitive. Let $a$ and $b$ be any two nonzero elements of $G$ satisfying $h(a) \leqq h(b)$ and let $A$ and $B$ denote the pure subgroups of $G$ generated by $a$ and $b$ respectively. By Lemma 3.9, some $f \in \operatorname{Hom}_{z}(A, B)$ maps $a$ to $b$. By $[5$, p.178], there is a projection $g$ of $G$ onto $A$. Now $f g \in F(G)$ sends $a$ to $b$, so $F(G)$ is fully two-fold transitive.

(2) implies (1). By Remark 3.7 (4) and Corollary 1.7, $G$ is homogeneous and every pure subgroup $A$ of rank one is a quasi-summand; it will be sufficient to show that $A$ is in fact a direct summand [5, p.178]. Write $G \doteq A+C$ with $C$ pure in $G$. By $[1, \mathrm{p} .96]$,

$$
G=B+C
$$

with $B$ isomorphic to $A$ via some map $f$; let $g$ be the projection of $G$ onto $B$. Pick a nonzero element $a \in A ; h(a)=h\left(f^{-1} a\right)$ and height is unambiguous since all relevant groups are pure subgroups of $G$. By hypothesis, some $r \in F(G)$ maps $a$ to $f^{-1} a$. Let $s=f g r ; s a=a$. $\{c \in G: s c=c\}$ is a nontrivial pure subgroup of $G$ contained in $A$ and so equals $A$, i.e., $s$ is an idempotent.

(2) and (3) are equivalent by Remark 3.7 (4) and Theorem 2.5.

(1) implies (4). Since (1) implies (3), it will be enough to prove that $F(G)$ is dense in the finite topology of $E(G)$. Let $f$ be any endomorphism of $G$ and let $a_{1}, \cdots, a_{n}$ be arbitrary elements of $G$. Now $a_{i}, f a_{i}, i=1, \cdots, n$, are all contained in some direct summand 
of finite rank. If $g$ is a projection associated with this summand, $g f \in F(G)$ is in the open neighborhood of $f$,

$$
\left\{h \in E(G): h a_{i}=f a_{i}, i=1, \cdots, n\right\} .
$$

(4) implies (1). It will be sufficient to see that any pure subgroup $A$ of finite rank in $G$ is a direct summand [5, p.178]. By density, some $f \in F(G)$ leaves $A$ invariant because the identity map does. Let $B$ denote the pure subgroup of $G$ generated by $f G$; by hypothesis $B$ is completely decomposable and $A$ is a direct summand of $B$ by [5, p.178]. If $g$ projects $B$ onto $A$ then $g f$ projects $G$ onto A.

REMARK 3.11. Full two-fold transitivity cannot be strengthened in the following sense. Given $a_{1}, a_{2}$ independent in $G$ and $b_{1}, b_{2}$ arbitrary in $G$ such that $h\left(a_{i}\right) \leqq h\left(b_{i}\right)$, in general there is no endomorphism mapping $a_{i}$ to $b_{i}, i=1,2$. Furthermore in Theorem 3.10 (3), the complete decomposability of pure subgroups of finite rank is essential, as the following discussion indicates. Let $K$ be any subfield of the $p$-adic number field $F_{p}$ and let $R=K \cap J_{p}, J_{p}$ the subring of $p$-adic integers; $R$ is a pure subring of $J_{p}$ and so is indecomposable [5, p.150]. By standard arguments [5, p.212], $E(R)=R$, i.e., every endomorphism of the additive group of $R$ is induced by ring multiplication. Now it is easy to see that $E(R)$ is fully one-fold transitive, for if $a$ and $b$ are nonzero elements of $R, a=p^{m} u, b=p^{n} v$ with $u$ and $v$ units in $J_{p}[6, \mathrm{p} .225]$ and hence in $R$ by purity; also

$$
u^{-1} \in R=K \cap J_{p} \text {. }
$$

Now $h(a) \leqq h(b)$ if and only if $m \leqq n[6, \mathrm{p} .225]$, so if $m \leqq n$,

$$
p^{n-m} v u^{-1} \in R=E(R)
$$

maps $a$ to $b$; otherwise $v u^{-1}$ maps $a$ to $p^{m-n} b$. Thus $E(R)$ is fully one-fold transitive. In particular for $K$ an algebraic number field [6, p.229], $E(R)=F(R), F(R)$ is fully one-fold transitive but not (fully) two-fold transitive, and $R$ is homogeneous but not (quasi-) separable.

\section{REFERENCES}

1. R. A. Beaumont and R. S. Pierce, Torsion-free rings, Illinois J. Math., 5 (1961), 61-98.

2. Torsion free groups of rank two, Memoirs Amer. Math. Soc., 38 (1961),

3. R. A. Beaumont and H. S. Zuckerman, A characterization of the subgroups of the additive rationals, Pacific J. Math., 1 (1951), 169-177.

4. E. F. Cornelius, Jr., Note on quasi-decompositions of irreducible groups, Proc. Amer. Math. Soc., 26 (1970), 33-36. 
5. L. Fuchs, Abelian Groups, Budapest, 1960.

6. N. Jacobson, Lectures in Abstract Algebra, vol. 3, Princeton, 1964.

7. N. Jacobson, Structure of Rings, Amer. Math. Soc. Colloquium Publications, vol. 37, 1956.

8. J. D. Reid, On quasi-decompositions of torsion free abelian groups, Proc. Amer. Math. Soc., 13 (1962), 550-554.

9. - On the ring of quasi-endomorphisms of a torsion-free group, Topics in Abelian Groups, Chicago, 1963, 51-68.

Received February 17, 1970. This paper is a revision of part of the author's doctoral thesis written under Professor R. A. Beaumont at the University of Washington, Seattle, Washington.

WAYNe State UNIVERSITY 



\title{
PACIFIC JOURNAL OF MATHEMATICS
}

\author{
EDITORS
}

\author{
H. SAMELSON \\ Stanford University \\ Stanford, California 94305 \\ C. R. HOBBY \\ University of Washington \\ Seattle, Washington 98105
}

J. DugundJI

Department of Mathematics

University of Southern California

Los Angeles, California 90007

RICHARD ARENS

University of California

Los Angeles, California 90024

\section{ASSOCIATE EDITORS}
E. F. BeCKenBaCH
B. H. NeUMANN
F. WOLF
K. Yoshida

\section{SUPPORTING INSTITUTIONS}

\author{
UNIVERSITY OF BRITISH COLUMBIA \\ CALIFORNIA INSTITUTE OF TECHNOLOGY \\ UNIVERSITY OF CALIFORNIA \\ MONTANA STATE UNIVERSITY \\ UNIVERSITY OF NEVADA \\ NEW MEXICO STATE UNIVERSITY \\ OREGON STATE UNIVERSITY \\ UNIVERSITY OF OREGON \\ OSAKA UNIVERSITY
}

\author{
UNIVERSITY OF SOUTHERN CALIFORNIA \\ STANFORD UNIVERSITY \\ UNIVERSITY OF TOKYO \\ UNIVERSITY OF UTAH \\ WASHINGTON STATE UNIVERSITY \\ UNIVERSITY OF WASHINGTON \\ AMERICAN MATHEMATICAL SOCIETY \\ NAVAL WEAPONS CENTER
}

The Supporting Institutions listed above contribute to the cost of publication of this Journal, but they are not owners or publishers and have no responsibility for its content or policies.

Mathematical papers intended for publication in the Pacific Journal of Mathematics should be in typed form or offset-reproduced, (not dittoed), double spaced with large margins. Underline Greek letters in red, German in green, and script in blue. The first paragraph or two must be capable of being used separately as a synopsis of the entire paper. The editorial "we" must not be used in the synopsis, and items of the bibliography should not be cited there unless absolutely necessary, in which case they must be identified by author and Journal, rather than by item number. Manuscripts, in duplicate if possible, may be sent to any one of the four editors. Please classify according to the scheme of Math. Rev. Index to Vol. 39. All other communications to the editors should be addressed to the managing editor, Richard Arens, University of California, Los Angeles, California, 90024.

50 reprints are provided free for each article; additional copies may be obtained at cost in multiples of 50 .

The Pacific Journal of Mathematics is published monthly. Effective with Volume 16 the price per volume (3 numbers) is $\$ 8.00$; single issues, $\$ 3.00$. Special price for current issues to individual faculty members of supporting institutions and to individual members of the American Mathematical Society: $\$ 4.00$ per volume; single issues $\$ 1.50$. Back numbers are available.

Subscriptions, orders for back numbers, and changes of address should be sent to Pacific Journal of Mathematics, 103 Highland Boulevard, Berkeley, California, 94708.

PUBLISHED BY PACIFIC JOURNAL OF MATHEMATICS, A NON-PROFIT CORPORATION

Printed at Kokusai Bunken Insatsusha (International Academic Printing Co., Ltd.), 270, 3chome Totsuka-cho, Shinjuku-ku, Tokyo 160, Japan. 


\section{Pacific Journal of Mathematics}

\section{Vol. 39, No. $3 \quad$ July, 1971}

William O'Bannon Alltop, 5-designs in affine spaces ................... 547

B. G. Basmaji, Real-valued characters of metacyclic groups ................. 553

Miroslav Benda, On saturated reduced products....................... 557

J. T. Borrego, Haskell Cohen and Esmond Ernest Devun, Uniquely representable semigroups. II.......................................

George Lee Cain Jr. and Mohammed Zuhair Zaki Nashed, Fixed points and stability for a sum of two operators in locally convex spaces ....................

Donald Richard Chalice, Restrictions of Banach function spaces ...............

Eugene Frank Cornelius, Jr., A generalization of separable groups ..............

Joel L. Cunningham, Primes in products of rings ......................

Robert Alan Morris, On the Brauer group of $Z$.

593

603

615

David Earl Dobbs, Amitsur cohomology of algebraic number rings ...............

Charles F. Dunkl and Donald Edward Ramirez, Fourier-Stieltjes transforms and

weakly almost periodic functionals for compact groups ...................

Hicham Fakhoury, Structures uniformes faibles sur une classe de cônes et

d'ensembles convexes ......................................

Leslie R. Fletcher, A note on C $\theta \theta$-groups.

Humphrey Sek-Ching Fong and Louis Sucheston, On the ratio ergodic theorem for

semi-groups............................................

James Arthur Gerhard, Subdirectly irreducible idempotent semigroups ...........

Thomas Eric Hall, Orthodox semigroups.....................

Marcel Herzog, $C \theta \theta$-groups involving no Suzuki groups ..........

669

687

John Walter Hinrichsen, Concerning web-like continua ..........

691

Frank Norris Huggins, A generalization of a theorem of F. Riesz.

695

Carlos Johnson, Jr., On certain poset and semilattice homomorphisms

703

Alan Leslie Lambert, Strictly cyclic operator algebras ...........

717

Howard Wilson Lambert, Planar surfaces in knot manifolds . . .

727

Robert Allen McCoy, Groups of homeomorphisms of normed linear spaces ....... 735

T. S. Nanjundiah, Refinements of Wallis's estimate and their generalizations ...... 745

Roger David Nussbaum, A geometric approach to the fixed point index .......... 751

John Emanuel de Pillis, Convexity properties of a generalized numerical range .... 767

Donald C. Ramsey, Generating monomials for finite semigroups ....

783

William T. Reid, A disconjugacy criterion for higher order linear vector differential equations...

Roger Allen Wiegand, Modules over universal regular rings...

Kung-Wei Yang, Compact functors in categories of non-archimedean Banach

spaces.

R. Grant Woods, Correction to: "Co-absolutes of remainders of Stone-Čech compactifications".

Ronald Owen Fulp, Correction to: "Tensor and torsion products of

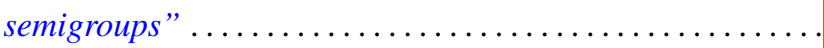

Bruce Alan Barnes, Correction to: "Banach algebras which are ideals in a banach 